\title{
Professional Association News
}

Les ingénieurs forestiers engagés à poursuivre I'intensification de l'aménagement forestier

À la lumière des informations livrées par les conférenciers et les scientifiques lors de leur congrès annuel, les ingénieurs forestiers ont réaffirmé leur engagement en faveur la poursuite de l'intensification de l'aménagement des forêts québécoises. C'est sur cette orientation que se sont laissés les ingénieurs forestiers au sortir de leur $81^{\mathrm{e}}$ congrès annuel qui se tenait la semaine dernière à Trois-Rivières, sous le thème "Aménager intensivement : où et comment?».

Ainsi, autant lors du premier bloc de conférences du jeudi matin qu'à l'occasion de la sortie sur le terrain de l'aprèsmidi ou des discussions en plénière tenues le lendemain, le même fil conducteur s'est dégagé de la réflexion collective : la plus grande diversité des utilisations du milieu forestier mènera droit à l'intensification des travaux d'aménagement forestier sur des portions plus ciblées du territoire. En effet, les ingénieurs forestiers croient qu'il sera nécessaire de protéger des portions importantes de ce territoire forestier à des fins de conservation de la biodiversité, tout en compensant par la pratique d'une foresterie plus intensive sur d'autres portions de ce même territoire, de façon à répondre aux besoins de l'industrie forestière en matière d'approvisionnement. Entre les deux, une foresterie s'inspirant de la dynamique des forêts naturelles devra être appliquée sur une vaste partie du territoire. L'objectif sera donc de produire plus de bois sur une moins grande superficie, tout en bénéficiant de plus grandes portions d'aires protégées. Pour mieux répondre aux divers usages, la définition d'affectations du territoire forestier à l'échelle régionale devra être sérieusement envisagée.

Soulignons que le choix de cette thématique se situe dans la perspective de l'adoption, en 2002, d'une politique québécoise sur le rendement accru tel qu'annoncé dans la Loi 136 modifiant le Loi sur les forêts, adoptée en juin dernier. Les ingénieurs forestiers ont ainsi voulu établir les bases de leur position en regard de la consultation annoncée par le gouvernement.

Selon la présidente-directrice générale de l'Ordre, madame Johanne Gauthier, ing.f., pour parvenir à intensifier l'aménagement forestier, toutes les méthodes sylvicoles devront être mises à contribution soit: l'établissement de la régénération naturelle, le reboisement lorsque nécessaire, l'éclaircie et l'éducation des jeunes forêts, la protection contre les insectes et les maladies, etc. et ce, tant pour les forêts feuillues que pour les forêts mélangées ou résineuses.

Le potentiel du reboisement en tant que moyen d'augmenter la productivité des sites a de nouveau été reconnu, tout comme celui de la ligniculture, c'est-àdire la culture intensive d'arbres en vue d'obtenir le maximum de bois dans un minimum de temps. Ce dernier modèle se rapproche des méthodes agricoles et vise la récolte des arbres après $15 \mathrm{ou}$ 20 ans et il pourrait s'appliquer à certains endroits très ciblés.

Pour parvenir à mettre en place un tel projet à l'échelle du Québec, des conditions essentielles de réussite ont été identifiées :

- Une définition de l'aménagement forestier intensif devra être élaborée, ainsi que des objectifs de production à l'échelle du Québec.

- L'acceptation d'un tel projet par le public et les communautés devra être assurée; pour ce faire, des processus régionaux de concertation et de définition des objectifs devront être établis, au cours desquels les enjeux sociaux, économiques et environnementaux seront soupesés; les objectifs de biodiversité devront alors être pris en compte

- Les travaux en forêt devront être exécutés par une main-d'œuvre qualifiée, ayant bénéficié d'une formation préalable de haut niveau; la mise sur pied de programmes de formation et d'accréditation des travailleurs forestiers favorisera à la fois la qualité des travaux, la stabilité de la main-d'œuvre et la présence d'une relève adéquate.

- L'état devra assumer une part importante du financement d'un programme provincial d'intensification de l'aménagement forestier, mais des incitatifs fiscaux devraient également être examinés de façon à favoriser l'apport d'investissements privés.

- Les diverses vocations du territoire devront être stables dans le temps, de façon à ce que les forêts aménagées demeurent disponibles pour la récolte forestière, le moment venu.

- Le reboisement et la ligniculture devront être alimentés par un programme d'amélioration des jeunes plants qui permettra non seulement l'augmenta-

\section{Assurances responsabilité professionnelle pour les membres de l'Institut en consultation}

La police d'assurances responsabilité professionnelle de l'Institut gérée par LMS Prolink Ltd Insurance Brokers vous fournira une protection de responsabilité légale en tant que tierce partie suite à une erreur reconnue, une erreur imputée, une omission ou un geste de négligence sous: Perte financière et vos coûts légaux.

Pour plus d'information, veuillez communiquer avec Rick Barnard, c.a., CAIB au 416595-7484 ou au 1-800-663-6828, courriel: rickb@lms.ca. Pour un formulaire d'adhésion, veuillez communiquer avec le bureau national de l'Institut au tél.: 613-2342242, télécopieur: 613-234-6181, courriel:cif@cif-ifc.org

Police d'assurances groupe pour les membres de l'Institut forestier du Canada

L'Institut est heureux de fournir à ses membres un plan d'assurances groupe. Cette police est souscrite auprès de LMS Prolink. Elle offre:

- Assurance-vie et assurance décès et compensations en cas de perte de membre

- Compensation hebdomadaire pour perte de revenus

- Compensation pour incapacité à long terme

De plus, elle comprend une programme d'assurances pour les personnes à charge des membres:

- Programme d'aide aux membres "Connections"

- Assurance-santé étendue

- Assurance soins dentaires

Pour plus d'information, veuillez communiquer avec LMS Prolink Ltd. au 1-800-6636828, 416-595-7484, courriel info@lms.ca 
tion de la croissance des arbres mais également le développement de propriétés chimiques ou mécaniques qui répondront aux marchés où les bois seront destinés.

- Des processus efficaces et transparents de communication devront être mis en place par l'ensemble des acteurs du milieu forestier, dont l'Ordre, de façon à bien expliquer aux différents acteurs et au grand public, les tenants et aboutissants de l'aménagement forestier intensif. À cet effet, les ingénieurs forestiers ont convenu qu'ils gagneront à travailler de pair avec les autres professionnels intéressés par le milieu forestier de façon à élargir les visions et à uniformiser le vocabulaire.

Tout ceci s'inscrit dans une dynamique internationale où les clients acheteurs des produits forestiers québécois sur les marchés internationaux expriment des attentes grandissantes en termes de protection de l'environnement. En même temps, l'industrie québécoise fait face à de nouveaux pays compétiteurs dont les approvisionnements en bois proviennent majoritairement de forêts aménagées intensivement. Les ingénieurs forestiers croient que le Québec devra se démarquer en misant sur la qualité des produits forestiers qu'il pourra offrir sur ces marchés.

En choisissant ce thème de congrès, les ingénieurs forestiers ont voulu faire le point sur les façons de répondre aux attentes grandissantes de la société en termes de protection des milieux et de maintien de l'apport économique et social des ressources forestières. Comme acteurs principaux intervenant dans cet écosystème, ils ont à traduire le concept d'aménagement forestier durable en actions concrètes sur le terrain.

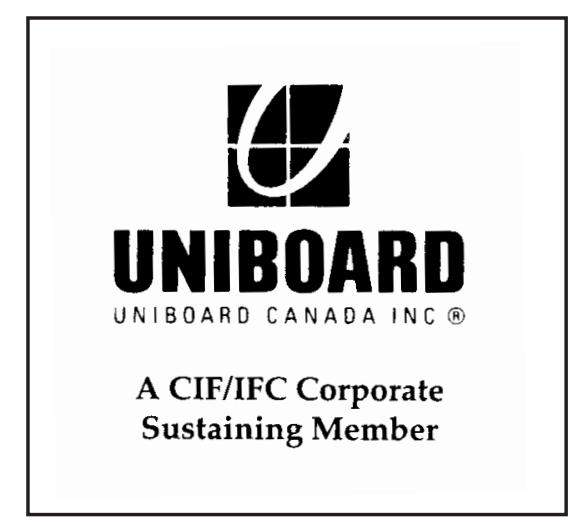

\section{L'Ordre des ingénieurs forestiers du québec décerne ses distinctions pour l'année 2001}

L'Ordre des ingénieurs forestiers du Québec a décerné ses distinctions pour l'année 2001, lors du banquet de clôture de son congrès annuel qui se tenait vendredi soir dernier à Trois-Rivières, sous le thème "Aménager intensivement : où et comment?».

Comme à l'habitude, l'Ordre a procédé à la remise de trois distinctions, soit la « Médaille d'honneur de l'Ordre », « l'Ingénieur forestier de l'année » ainsi que la distinction « Henri-Gustave-Jolyde-Lotbinère ». Cette dernière reconnaissance est instituée pour permettre à l'Ordre de valoriser certains services ou contributions exceptionnels à la cause forestière, de la part de personnes externes à la profession.

La présidente-directrice générale de l'Ordre, Mme Johanne Gauthier, ing.f., a procédé à la remise de ces distinctions en présence de plus de deux cents congressistes. Les récipiendaires retenus par l'Ordre sont les suivants :

\section{La " Médaille d'Honneur De L'ordre "}

Cette reconnaissance est instituée pour permettre à l'Ordre de reconnaître les services exceptionnels rendus à la profession d'ingénieur forestier par un ou une de ses membres tout au long de sa carrière professionnelle.

Le choix du Bureau de l'Ordre s'est arrêté cette année sur monsieur René Doucet, ing.f.

\section{René Doucet, ing.f., Ph.D.}

L'Ordre a retenu la candidature de monsieur Doucet en raison de l'importance de sa contribution à l'avancement et à la diffusion de connaissances en sylviculture tout au cours de sa carrière scientifique et professionnelle. En effet, monsieur Doucet jouit d'une réputation enviable au sein des communautés scientifique et forestière québécoises à titre de spécialiste de la sylviculture. Il a développé une expertise unique dans la culture du pin gris, du peuplier faux-tremble et de l'épinette noire et les résultats de ses travaux de recherche ont généré de multiples applications sur le terrain.

C'est ainsi que ses recherches sur la régénération de l'épinette noire, débutées en 1983, ont mené, au début des années 1990, à l'acceptation des marcottes et des tiges dites « supprimées » à titre de régénération forestière viable et satis-

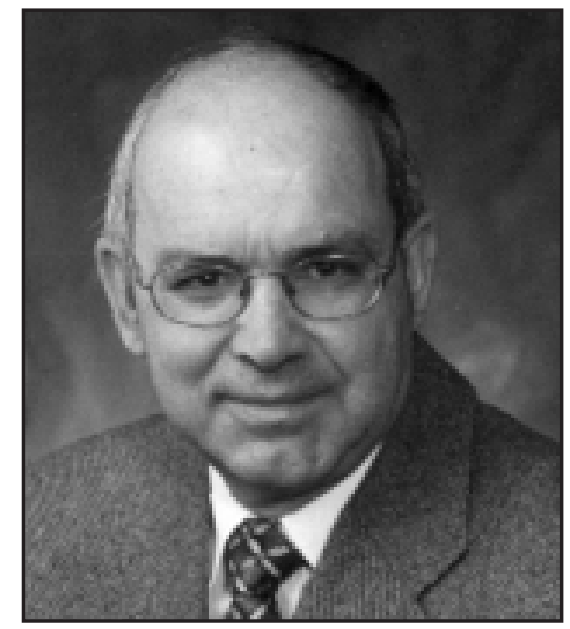

René Doucet

faisante. Par ricochet, les résultats de ces recherches ont fait évoluer de façon significative la gestion des forêts québécoises vers l'adoption de la régénération naturelle comme principale méthode de régénération des forêts d'épinette. Le ministère des Ressources naturelles du Québec en a même fait l'une des principales assises de sa Stratégie de protection des forêts.

Les recherches sur le marcottage de l'épinette noire ont amené monsieur Doucet à élargir le champ des connaissances sur la sylviculture de cette essence, en apportant notamment des éclairages sur l'éclaircie pré commerciale, la régénération des sols minces de la CôteNord et l' « ensapinage » des peuplements d'épinette noire.

Monsieur Doucet a aussi réalisé d'importantes activités de recherche en sylviculture du peuplier et du pin gris, qui ont fait l'objet de multiples présentations. Ces activités se sont déroulées en même temps qu'il assumait la gestion de toute une équipe de recherche.

M. Doucet est diplômé de la Faculté de foresterie et de géomatique de l'Université Laval en 1966. Il a obtenu une maîtrise de la même institution en 1968, puis s'est inscrit à State University of New York, à Syracuse, qui lui décernera un doctorat en 1974. Il a débuté sa carrière dans l'industrie forestière et œuvré ensuite au ministère du Tourisme, au Centre de recherches forestières des Laurentides, puis à l'Université Laval. Il a ensuite exercé sa profession au ministère des Ressources naturelles du Québec de 1969 à 1995, à la Direction de la recherche forestière; d'abord comme chargé de recherches et responsable de 
la Division Recherche-Développement; puis comme chef de la Division en Recherche-Développement en sylviculture des forêts naturelles; et enfin à titre de chef de la Division en RechercheDéveloppement en écologie et sylviculture des forêts naturelles et de chargé de recherches jusqu'en 1995.

René Doucet est une personne intègre, tenace et perspicace, dotée d'un excellent esprit d'analyse et de synthèse. Il est reconnu pour sa compétence, sa disponibilité, son dévouement et sa grande humilité. Il possède d'autre part une vision globale de la forêt et constitue un collaborateur et un vulgarisateur incomparable, d'une grande rigueur scientifique et attentif aux besoins de la collectivité.

Tout au long de sa carrière, il s'est assuré du transfert aux utilisateurs des nouvelles connaissances acquises par des contacts personnels, des visites de terrain et par la participation à une dizaine de comités permanents ou ad hoc. Ses nombreuses publications scientifiques $(84$ à son actif) et les multiples invitations à présenter ses travaux à l'extérieur du Québec témoignent de sa renommée.

Sa participation à l'organisation de plusieurs événements ainsi que son implication dans l'enseignement et la direction d'étudiants gradués illustrent également le rayonnement de ses actions. Il a de plus été actif au niveau professionnel en s'impliquant activement dans les activités de l'Institut forestier du Canada et de l'Ordre. Ainsi, depuis 1999, il agit à titre de représentant de l'Ordre au Bureau canadien d'agrément en foresterie. Compte tenu qu'il est maintenant à la retraite, l'Ordre est heureux de pouvoir compter sur son sens du professionnalisme.

Par cette sélection, l'Ordre tient donc à souligner la diversité de sa carrière, ses nombreuses réalisations scientifiques et professionnelles ainsi que son importante contribution au rayonnement de la profession.

\section{" L'ingénieur forestier de I'année"}

Le titre d" "Ingénieur forestier de l'année » est décerné à un membre pour souligner sa contribution exceptionnelle au développement et à la promotion de la profession d'ingénieur forestier. Le ou la récipiendaire devra avoir accompli un acte méritoire remarquable dans les sphères d'activités de la profession au cours des 24 derniers mois. Il pourra s'agir d'une contribution personnelle exemplaire dans les domaines scientifique, culturel, humanitaire social ou des communications.

Le choix du Bureau de l'Ordre s'est arrêté cette année sur monsieur Marc Plante, ing.f.

\section{Marc Plante, ing.f.}

Par cette distinction, l'Ordre tient à souligner la contribution exceptionnelle de monsieur Marc Plante à l'organisation du Forum Forêt-Faune tenu à deux reprises à Jonquière, d'abord en 1997 puis en novembre 1999. La seconde édition de cet événement était organisée conjointement par l'Ordre des ingénieurs forestiers du Québec, l'Association des biologistes du Québec, la Société de la Faune et des Parcs du Québec, le ministère des Ressources naturelles - Forêt Québec, le Consortium de recherche sur la forêt boréale commerciale, le Cégep de St-Félicien et l'Université du Québec à Chicoutimi.

Ces colloques ont connu un franc succès (500 participants par colloque). Les communications scientifiques et les ateliers ont favorisé un rapprochement des utilisateurs forestiers et fauniques tout en permettant à chacun de faire connaître ses besoins et ses préoccupations. Ces colloques furent également l'amorce d'un dialogue plus formel entre les utilisateurs du milieu forestier et le Groupe Faune régional et ils ont facilité la mise en place d'un groupe régional sur la gestion intégrée des ressources.

L'implication de monsieur Plante dans l'organisation de deux colloques Forêt-Faune de 1997 et de 1999, avec l'Association des biologistes du Québec, a également favorisé un rapprochement de deux associations professionnelles à l'égard de la gestion des ressources du milieu forestier.

Il est important de souligner que c'est à titre bénévole que Marc Plante a pris l'initiative en 1996 de créer, avec Mme Nathalie Perron, biologiste, un nouvel événement visant à regrouper l'ensemble des intervenants et des utilisateurs des milieux forestier et faunique. Conscients de l'importance de renforcer les mesures de concertation dans ce secteur, ils ont su mobiliser l'ensemble des intervenants régionaux et mettre sur pied un comité organisateur multidisciplinaire.

Loin d'être un événement local, le Forum Forêt-Faune a même rayonné au-delà des limites du Québec, puisque

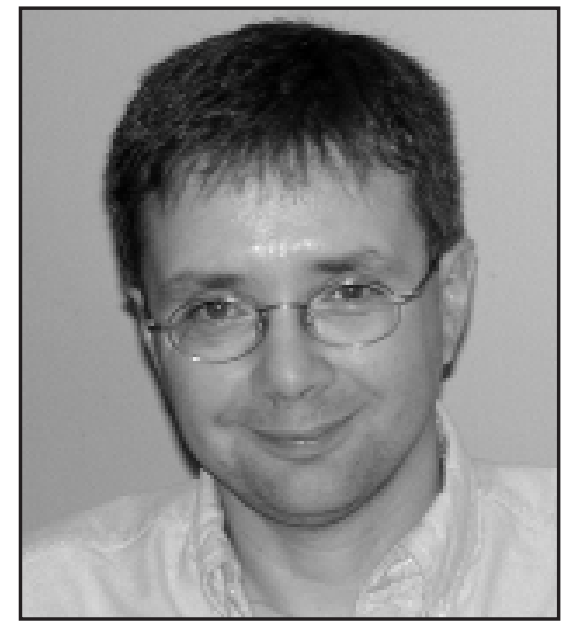

Marc Plante

des représentants du Nouveau-Brunswick ont assisté à l'événement. Sans contredit, les deux éditions du Forum ont constitué un lieu de rencontres et d'échanges pour des dizaines d'ingénieurs forestiers, de biologistes, d'étudiants universitaires, de pourvoyeurs, de gestionnaires de zecs, d'industriels forestiers et d'autochtones. Ce forum a aussi contribué à la formation et à la sensibilisation de centaines d'étudiants en foresterie et en biologie, lesquels seront les décideurs de demain. La création d'un site Internet francophone, conçu spécialement pour l'événement, a favorisé une plus large diffusion de l'information. L'imposante revue de presse déposée avec la candidature montre l'impact important de l'événement.

Marc Plante a reçu son diplôme en génie forestier de la Faculté de foresterie et de géomatique de l'Université Laval en 1985. Il débute sa carrière à la Société de conservation du Saguenay - Lac-SaintJean, pour ensuite exercer sa profession en 1986 au ministère des Ressources naturelles du Québec, à l'unité de gestion de Chibougamau, comme responsable de l'analyse et de la planification. Il a poursuivi sa carrière à Forêt Québec, à Jonquière, à partir de 1992 en tant que responsable de la planification forestière.

Il a agi à titre de président du comité sur l'assurance-responsabilité professionnelle de l'Ordre et il a été membre du comité de formation continue de l'Ordre en 1996 et 1997. De plus, il a été administrateur régional au sein du Bureau de l'Ordre de 1994 à 1997.

Les membres du comité soulignent l'apport de M. Plante au rayonnement de 
la profession d'ingénieur forestier et sa contribution particulière à l'avancement d'idées innovatrices en foresterie au cours des deux dernières années.

\section{La distinction " Henri-Gustave- Joly-De-Lotbinière "}

La distinction « Henri-Gustave-Jolyde-Lotbinière » est remise à une personne extérieure à la profession dont les actions auront fait progresser la cause forestière et contribué à l'avancement et au rayonnement de la profession.

L'Ordre a retenu cette année l'Association forestière de l'Abitibi-Témiscamingue comme récipiendaire de la distinction « Henri-Gustave-Joly-deLotbinière ».

\section{L'Association forestière de l'Abitibi-Témiscamingue}

L'Ordre décerne cette distinction à 1'Association forestière de l'AbitibiTémiscamingue (AFAT) pour souligner la pertinence et le succès du Circuit forestier touristique de l'Abitibi-Témiscamingue et du Nord-du-Québec (CTF) mis de l'avant par cette association. Cette initiative a été lancée en 1999, après trois ans de travail.

Le Circuit touristique forestier est présenté dans une brochure de qualité et il constitue une première du genre au Québec. Il est disponible dans les centre d'informations touristiques de la région. À partir de la description de la forêt elle-même, de son interprétation et de son histoire, en passant par la coupe, les travaux sylvicoles, la transformation du bois, la recherche et les produits dérivés, le CTF cherche à sensibiliser les visiteurs au rôle primordial que jouent les ressources du milieu forestier. Le Circuit touristique forestier s'étend sur un vaste territoire et il permet de créer des relations entre les différents sites, tout en donnant un sens commun à l'ensemble du milieu forestier régional, ce qu'un seul site n'arrive souvent pas à traduire en raison de la diversité de la problématique du milieu.

Cet important outil d'information, d'éducation et de sensibilisation du grand public au milieu forestier comble différents besoins dont celui pour les gens du milieu forestier d'expliquer leurs interventions, lesquelles ont fait l'objet d'un certain questionnement au cours des dernières années. Il répond également au besoin du public d'être mieux renseigné sur ce qui se passe réellement en forêt.

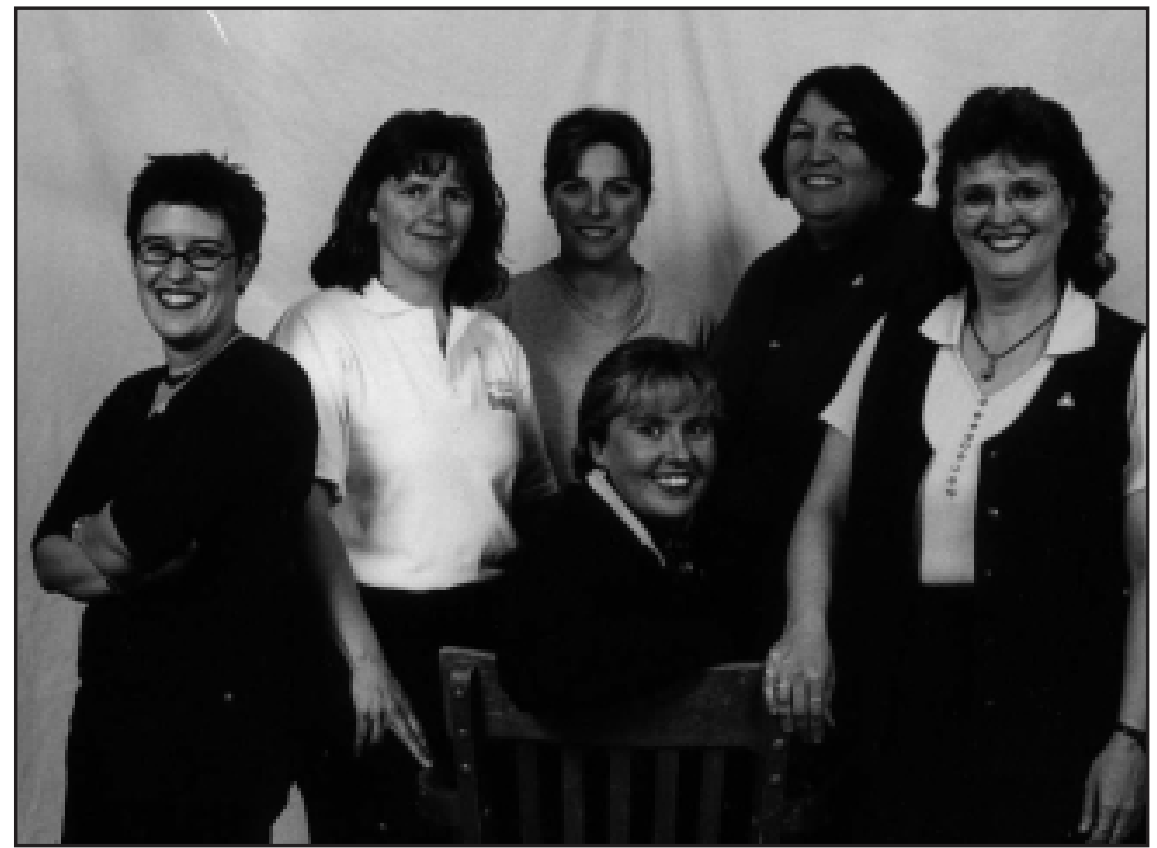

Le personnel de l'AFAT*: Mélanie Arcand, agente de communication; Lise Trudel, responsable du Coin de la carte et agente de bureau; Isabelle Reny, directrice générale; Nancy Fortier, chef-naturaliste au Centre éducatif forestier du lac Joannès; Linda St-Lovis, directrice générale adjointe; et Jacinthe Langevin, secrétaire comptable. Personnel présent au moment où le Circuit est né et où il a été lancé en 1999-2000.

Le CTF en est maintenant à sa troisième année d'existence et il a pris de l'ampleur depuis des débuts. Le projet était et est encore réalisé de concert avec de nombreux intervenants du milieu forestier et touristique, dont notamment les entreprises forestières régionales. Parce qu'il rend accessible l'ensemble des informations reliées à la gestion de l'environnement forestier, le CTF s'inscrit dans un mouvement tout à fait mobilisateur de développement durable.

Le CTF se démarque également parce qu'il bonifie l'activité touristique de façon nouvelle et différente autour de la forêt et c'est ce qu'a démontré le bilan de la première saison d'activités : les visiteurs ont apprécié se faire expliquer les travaux et voir la régénération abondante sur le territoire. Ils se sont dits rassurés pour l'avenir. Quant aux organisateurs, ils ont constaté qu'il est important de parler de l'aspect dynamique de la forêt et de son évolution. Le CTF contribue à valoriser les travailleurs et les différents métiers forestiers ainsi qu'à développer le sentiment d'appartenance des gens aux ressources forestières et à leur région.

L'Association forestière de l'AbitibiTémiscamingue est un organisme qui a pour mission de promouvoir auprès de tous les intervenants de la région l'ensemble des utilisations du milieu forestier ainsi que les réalités sociale, économique, environnementale et culturelle qui s'y rattachent. L'AFAT regroupe plus de 750 membres et opère également le Centre éducatif forestier du lac Joannès, visité annuellement par plus de 7000 personnes.

Le Circuit forestier touristique contribue certainement à l'avancement et au rayonnement de la profession au-delà des limites de la région. Bon nombre d'ingénieurs forestiers contribuent au succès du projet et bon nombre saluent cette initiative qui englobe l'ensemble des champs d'activités de la profession. Les membres du Comité des distinctions de l'Ordre ont également tenu à souligner la grande qualité du guide décrivant le Circuit touristique déposé avec la candidature. Ils considèrent une telle démarche comme un coup de maître qui risque d'inspirer d'autres régions du Québec et d'ailleurs.

Source: Pierre Breton, ing.f. Directeur des communications Tél.: (418) 6502411 Fax: (418) 650-2168, Courriel : pierre.breton@oifq.com

Visitez notre site Internet : www.oifq.com 


\section{New Brunswick Forest Technicians Association}

At 8:05 PM on Sept. 22, 2001 fall fell. Summer was over whether we had all our summer work done or not. For some in the Silviculture or fiber-producing fields it was a good summer with few days lost to rain, though some fire restriction did reduce production. For those in the fire suppression field it was a somewhat busier year then the past few. To your Board members it was a successful summer. We have started some programs to benefit the NBFTA through education and public awareness. The summer started off with a joint Continuing Education Program with the RPF, CIF and CFS that gave us an insight into hardwood management. Early in June we had the opportunity to change some of New Brunswick Legislation under the Clean Water Act. Later in the summer we got the good news that the Tree House received and accepted our Region 4 application for the "Adopt the Highway Program" for the Rusagonis area. An NBFTA first but, I hope, not the last. Not to be out done, Kim Mann worked all summer with the Sylvicon 2002 committee to finalize an outstanding program for February 2002 plus secure the NBFTA as the 2003 host partner. To close out the year with something to look forward to, a joint Newsletter with the Tree House, RPF and CIF and the NBFTA may be in the future. If we can pull this together it will cap-off a very successful year for the NBFTA.

That is all the good news, and we all know that with the good comes a little bad. Our membership roll indicates we are 350 plus strong. What it does not show is that close to 100 members have not paid their 2001 annual dues. Our constitution and bylaws are very clear on payment of dues. They must be paid 30 days after our Annual General Meeting. If not paid, the member has one year to pay but must pay a $25 \%$ penalty. If that member is a CFT then they can lose their designation as a CFT and will have to write their certification exam over. So, if you have not paid yet, please send your dues in, it is not much but yet it makes a difference to your organization. I hope your fall is as profitable as the past summer. If you have any question, problems or would like to get more involved with the NBFTA please contact your regional representative or me. We are always willing and able to help you out any way we can. Remember reserve Feb 22, 2002 for Sylvicon and your Annual General Meeting at the Fredericton Inn in Fredericton.

Walter Bidlake, NBFTA President

\section{Joint sessions held across Alberta to discuss proposed new regulations}

The Alberta Registered Professional Foresters Association (ARPFA) and Alberta Forest Technologists Association (AFTA) have completed seven joint consultation sessions across the province, relating to proposed regulations for the new Regulated Forestry Profession Act.

The sessions were well attended and attracted 200 people (166 members and 34 non-members).

A record of all questions posed at the sessions is posted on both association websites and by the end of October it was expected a complete summary report would also be posted.

This completes the association consultation phase on the proposed regulations; Alberta Human Resources and Employment will soon begin its external consultations. This includes soliciting input from other regulated professions in the province, regulated professions in the province, regulated bodies in other provinces in the forestry sector to address the labor mobility provisions under the federal Agreement on Internal Trade, and other government departments. Further government review and endorsement of the regulations will be provided by the Standing Policy Committee on Education and Employment prior to ultimate approval by Cabinet.

Recently, several amendments have been reviewed and endorsed by government relating to the Health Professions Act (HPA). Since the new legislation governing foresters and forest technologists was originally drafted using the HPA as the template, it will be necessary to make some minor amendments to it as well. Please check the association websites for further details on these proposed amendments.

ARPFA registrar Doug Krystofiak says both associations remain eager to achieve proclamation of the Regulated Forestry Profession Act by spring of 2002.

For more information, contact either association and continue to monitor the association websites for any recent developments on regulating the forestry profession.

Contact: ARPFA at (780) 432-1177 or www.arpfa.org; AFTA at (780) 4321962 or www.arpfa.org/afta.

\section{Errors and Omissions Insurance for CIF/IFC Consultant Members}

CIF/IFC Errors \& Omissions policy administered by LMS Prolink Ltd. Insurance Brokers will provide you with coverage for third party legal liability resulting from an actual or alleged error, omission, or negligent act for: Financial Loss and your Legal costs.

For more information: please contact Rick Barnard C.A., CAIB at 416-5957484 or 1-800-663-6828, email: rickb@Ims.ca. For an application form, please contact the CIF/IFC National Office at Tel : 613-234-2242, Fax: 613-234-6181, Email: cif@cif-ifc.org

Group Benefits plan for CIF/IFC Members:

$\mathrm{CIF} / \mathrm{IFC}$ is pleased to provide a group benefits plan to their members. The plan is underwritten by LMS Prolink Ltd. It offers:

- Life Insurance and Accidental Death and

Dismemberment Benefits

- Weekly Income Benefit

- Long Term Disability Benefit

As well as optional Member Dependent Benefits:

- Member Assistance Program "Connections"

- Extended Health Care

- Dental

For more information, please contact LMS Prolink Ltd. 1-800-663-6828, 416595-7484, email info@lms.ca 